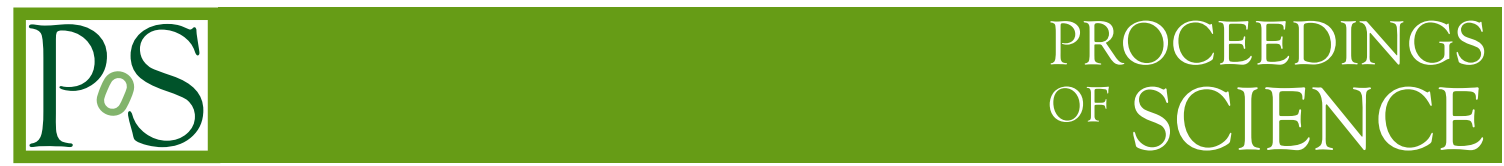

\title{
Muon Reconstruction and Identification in CMS
}

\author{
Marcin Konecki* \\ Institute of Experimental Physics, University of Warsaw, Poland \\ E-mail: marcin.konecki@gmail.com
}

\begin{abstract}
An event reconstruction at LHC is a challenging task. The Compact Muon Solenoid (CMS) is a general purpose experiment with a precise and redundant Muon System. A good muon reconstruction and identification is one of its design goals. In CMS muons are initially identified by dedicated Level-1 muon trigger while the precise reconstruction is completed at the High-Level Trigger and offline.

In this note the CMS Muon System is presented. The muon reconstruction algorithms both for event selection during triggering steps and offline, are described. The CMS experience with alignment of the Muon System using cosmic data are also given.
\end{abstract}

European Physical Society Europhysics Conference on High Energy Physics, EPS-HEP 2009,

July 16 - 222009

Krakow, Poland

* On behalf of the CMS Collaboration. 


\section{Introduction}

The main motivation for building the LHC is to explore the energy region up to a few $\mathrm{TeV}$, in a view of improving our understanding of the Electroweak Symmetry Breaking mechanism, searching for Supersymmetry and any kind of New Physics. Precise tests of the Standard Model are also important.

Since the predicted cross section for some discovery channels is very low, a large luminosity for LHC is mandatory. Thus selection of interesting events becomes challenging.

The Compact Muon Solenoid (CMS), currently ready for operation with the LHC beams, is a general purpose experiment for physics discoveries at the highest luminosities of LHC. The CMS design principles [1] include efficient lepton and photon selection and measurements following requirements of discovery physics. Within that, muons are especially important, due to a possibility of clean identification and possible low threshold during event selection.

\section{Muon System of the CMS detector}

The CMS Muon System [2] is located inside a return yoke of a superconducting solenoidal coil. A particle produced in the interaction point, in order to reach the Muon System, has to cross the Inner Tracking System (based on silicon tracking detectors), electromagnetic and hadronic calorimeters and the coil itself. Since large amount of material has to be passed, fraction of (interacting) particles other than muons is highly suppressed supporting identification of muons. The nearly 4 Tesla magnetic field inside the coil and the 1.8 Tesla in the return yoke ensures strong bending power allowing for a precise particle track measurement. Inside the CMS return yoke there are muon stations equipped with three types of muon detectors. The Drift Tube (DT) chambers are placed in the barrel part of CMS extending up to pseudorapidity $|\eta|<1.2$. The Cathode Strip Chambers (CSC) are placed in endcap disks. Their pseudorapidity coverage is $0.8<|\eta|<2.4$. The Muon System is completed with Resistive Plate Chambers (RPC) detector present both in barrel and endcap (limited up to $|\eta|<1.6$ in staged phase). RPC is dedicated for triggering, while DT and CSC are designed to provide precise measurement but also have triggering capabilities.

\section{Reconstruction steps}

There are several steps of muon selection and reconstruction in the CMS detector [3, 4]. Initially muons are reconstructed during Level-1 trigger decision using coarse-grained readout. The algorithms are implemented in partially programmable hardware logic. The Level-1 muon triggering is based on measurements of the track direction in muon stations and bending between muon stations. In turn, DT/CSC and RPC Level-1 triggers do not simply duplicate response but are complementary. In case of a successful Level-1 decision, further triggering steps are performed by the High-Level Trigger (HLT). HLT is implemented in a computer farm. In a Level-2 algorithm muons are reconstructed in the Muon System only using fine-grained information from DT and CSC. In a Level-3 algorithm, information from the Inner Tracker detector is additionally taken into account. Although these two algorithms are executed by the same computer program their distinction is useful, since the time needed to access data from various detector components is different. Both the 
Level-1 and the HLT reconstruction are performed online and optimized for rejection speed. The Level-1 trigger is supposed to reduce $40 \mathrm{MHz}$ LHC event rate to $100 \mathrm{kHz}$ which can be handled by HLT, which in turn reduces the event rate down to $\mathscr{O}(100 \mathrm{~Hz})$ as required by an event storage capabilities. The stored events can be reprocessed offline in purposes of dedicated analyzes with possibly improved calibration, alignment or optimized algorithms. The online and the offline reconstruction algorithms are similar.

\section{Muon Reconstruction algorithms [5]}

The muon reconstructed (both online and offline) in the Muon System is called a Stand-Alone Muon. The one supplemented with tracking detector measurements is a Global Muon.

The reconstruction of Stand-Alone Muons is preceded with Local Reconstruction. At first hits are reconstructed and combined within a chamber. In case of DT there are up to 8 measurements in $r-\varphi$ coordinate and 4 in $r-\theta$ one. In CSC up to 6 hits from planes are delivered. The straight line fitted across measurements provides a Track Segment. The Stand-Alone Muon reconstruction is based on the Kalman Filter Technique [6]. The trajectory is build starting from the innermost station. The forward pattern recognition (pre-filtering) collects compatible Track Segments which constrains the particle trajectory. After the trajectory is build a backward pattern recognition is performed with outside-in propagation. The trajectory is then updated using individual hits of Track Segments. Finally the trajectory at the innermost muon station is propagated to the nominal interaction region and, optionally, further constrained with a vertex condition. The online and offline Stand-Alone Muon reconstructions differ only in the definition of the initial trajectory state. The online algorithm uses information from the Level-1 trigger. The offline reconstruction performs a detector-wide analysis of segments. In latter case the seed kinematics is defined from a simple parameterization of bending between stations.

The Global Muon reconstruction does a combined fit of Stand-Alone Muon hits and selected hits from the tracker. A first step of reconstruction in tracker is a definition of a Region of Interest (ROI). It holds information about desired track position at vertex and its kinematics with tolerances. It is constructed using the Level-2 Stand-Alone Muon kinematics. For the offline reconstruction the tracks reconstructed from standard tracker reconstruction are accessed. These tracks are build with Combinatorial Track Finder beeing a Kalman Filter based algorithm consisting of hit-based seeding followed by inside-out pattern recognition, cleaning, fitting and backward fitting (smoothing). Only the tracker tracks compatible with previously defined ROI are selected for further matching. In the online reconstruction ROI resulted from a Stand-Alone Muon serves as an input for seed search and followed by Combinatorial Track Finder reconstruction. After reconstruction in the tracker trajectories of reconstructed tracks (from online or offline reconstruction) are compared with that of Stand-Alone Muon. In case of matching the combined re-fit is done and a Global Muon is constructed.

The performance of Stand-Alone and Global Muon reconstructions is summarized in Figures 1 and 2. In Figure 1 the reconstruction efficiency is shown as a function of pseudorapidity for selected transverse momenta. On average the efficiency of $95-98 \%$ is achieved except for regions where the Muon System has some geometrical gaps $(|\eta| \simeq 0.25,0.8,1.2,1.6)$. In Figure 2 the resolution of $\frac{q^{\text {rec }} / p_{T}^{\text {rec }}-q^{\text {gen }} / p_{T}^{\text {gen }}}{q^{\text {gen }} / p_{T}^{\text {gen }}}$ is shown. The $q$ and,$p_{T}$ stands for charge and transverse momentum 
(generated or reconstructed) respectively. One can see a significant improvement of the resolution for a fit exploring full system.
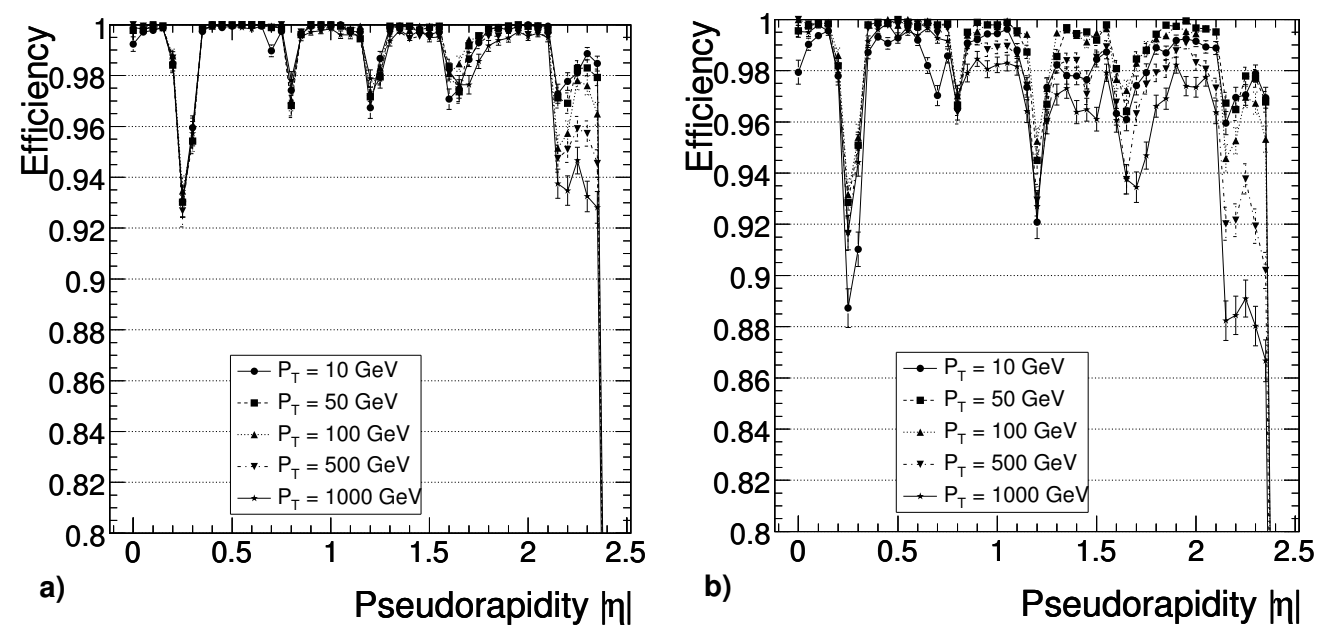

Figure 1: The efficiency of Stand-Alone (a) and Global (b) Muon reconstruction as the function of pseudorapidity for selected transverse momenta.
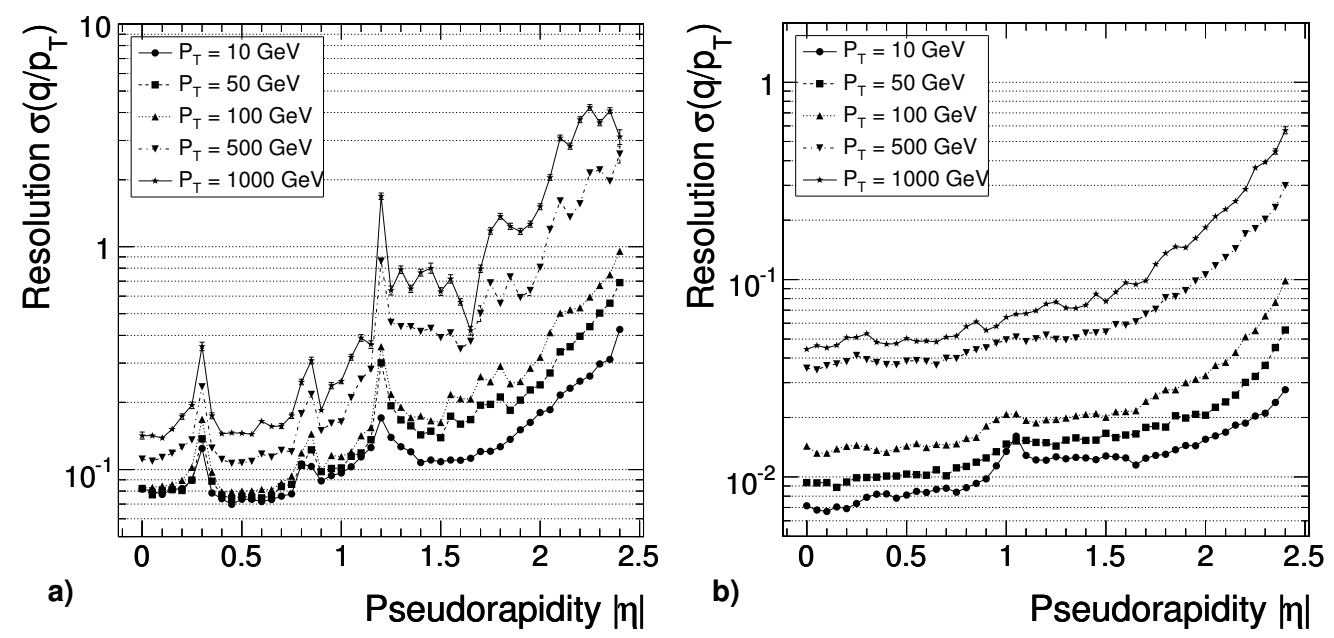

Figure 2: Resolution of $q / p_{T}$ for Stand-Alone (a) and Global (b) Muon reconstruction as the function of pseudorapidity for selected transverse momenta.

\section{First experience with cosmic data}

Since 2008 the baseline construction of the CMS detector has been finished. While waiting for the LHC beams, CMS is focusing on integration runs with cosmic muons. Among others this allows us to test muon reconstruction and inter-detector alignment. Since the structure of the CMS detector is not rigid deformations due to magnetic field, gravity (weight), temperature and humidity are expected. On the other hand, in order not to degrade the momentum measurements of a muon system a precision of better than $200 \mu \mathrm{m}$ is necessary. Thus CMS has developed alignment mechanisms using dedicated optical system and tracking. An example of such an integration exercise is 
shown in Figure 3. An impact of the allignment corrections on the muon reconstruction is clearly visiable.
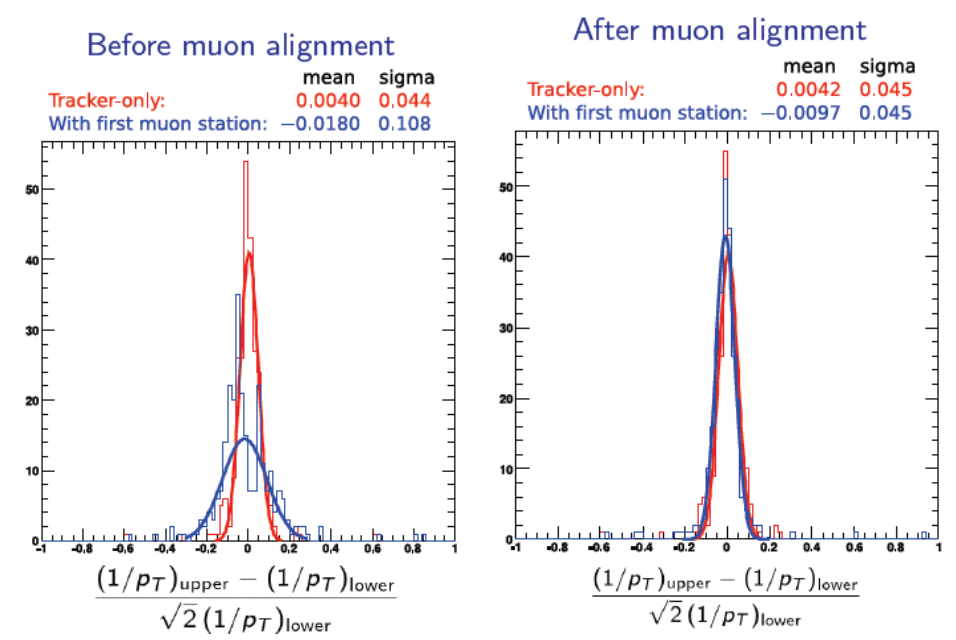

Figure 3: An example of muon system alignment exercise. Events with muons reconstructed independently in an upper and lower a part of detector are selected. The distribution of $1 / p_{T}$ difference between measurements is plotted. Muons are reconstructed using tracker-only data and tracker data plus first muon station measurements. The distributions are shown before and after alignment (left and right plots respectively). An obtained alignment precisions is equivalent to integrated LHC luminosity of $10 \mathrm{pb}^{-} 1$. Only tracks with $p_{T}>200 \mathrm{GeV} / c$ enters.

\section{Summary}

The CMS detector is a general purpose detector with a redundant muon system. The muon reconstruction procedure is well defined. The reconstruction sequence consists of reconstruction in Muon System only which is later combined with track reconstructed in the Inner Tracking Detector in order to achieve much better resolution. Currently CMS is focussing in integration which allow us to test for example alignment procedures. CMS is ready for the LHC beams.

\section{References}

[1] CMS Collaboration, CMS, The Compact Muon Solenoid, Letter of Intent, CERN/LHCC 92-3

[2] CMS Collaboration, CMS, The Muon Project, Technical Design Report, CERN/LHCC 97-32

[3] CMS Collaboration, CMS, The Trigger and Data Acquisition project, Volume I: The Level-1 Trigger, CERN/LHCC 2000-038

[4] CMS Collaboration CMS, The Trigger and Data Acquisition project, Volume II: Data Acquisition \& High-Level Trigger, CERN/LHCC 2002-26

[5] CMS Collaboration CMS Technical Design Report, Volume I: Detector Performance and Software, CERN/LHCC 2006-001.

[6] R. Fruhwirth, Nucl. Instrum. Meth., A262, 444-450 (1987). 